\title{
In Silico Hemostasis Modeling and Prediction
}

\author{
Dmitry Y. Nechipurenko*1,2,3 Aleksey M. Shibeko*2,3 Anastasia N. Sveshnikova ${ }^{1,2,3}$ \\ Mikhail A. Panteleev $1,2,3$ \\ ${ }^{1}$ Faculty of Physics, Lomonosov Moscow State University, \\ Moscow, Russia \\ ${ }^{2}$ Center for Theoretical Problems of Physicochemical Pharmacology \\ of the Russian Academy of Sciences, Moscow, Russia \\ ${ }^{3}$ Dmitry Rogachev National Research Center of Pediatric Hematology, \\ Oncology and Immunology, Moscow, Russia \\ Address for correspondence Prof. Mikhail A. Panteleev, 30 Srednyaya \\ Kalitnikovskaya Street, Moscow 109029, Russia \\ (e-mail: mapanteleev@yandex.ru).
}

Hämostaseologie 2020;40:524-535.

\begin{abstract}
Keywords

- computational biology

- mathematical modeling

- thrombosis

- hemostasis

Computational physiology, i.e., reproduction of physiological (and, by extension, pathophysiological) processes in silico, could be considered one of the major goals in computational biology. One might use computers to simulate molecular interactions, enzyme kinetics, gene expression, or whole networks of biochemical reactions, but it is (patho)physiological meaning that is usually the meaningful goal of the research even when a single enzyme is its subject. Although exponential rise in the use of computational and mathematical models in the field of hemostasis and thrombosis began in the 1980s (first for blood coagulation, then for platelet adhesion, and finally for platelet signal transduction), the majority of their successful applications are still focused on simulating the elements of the hemostatic system rather than the total (patho)physiological response in situ. Here we discuss the state of the art, the state of the progress toward the efficient "virtual thrombus formation," and what one can already get from the existing models.
\end{abstract}

\section{Introduction}

In silico methods play increasingly noticeable roles in all fields of biological sciences, at different scales and levels of research and development. Bioinformatics, molecular dynamics, and computer-assisted drug design have become widely used tools. In comparison with them, development of full-fledged in silico models of physiological and pathological processes lags behind: this "ultimate" goal of theoretical biology remains challenging even now. Still, the last decade witnessed their progress in many fields including that of hemostasis and thrombosis. ${ }^{1-7}$ Computer models of this type are being used for experimental planning, prediction and analysis in basic research, for drug target discovery, or to personalized diagnostics.

These authors contributed equally to this work.
The hemostatic response, as well as arterial thrombus formation, includes several steps beginning with platelet adhesion, activation, formation of aggregates, shape change, and secretion ( - Fig. 1A). ${ }^{8}$ These are usually grouped together as primary or platelet-dependent hemostasis. It is followed by secondary hemostasis, blood coagulation, that cements the initial platelet plug. ${ }^{9}$ We thus traditionally identify two essential parts of the hemostatic system. However, from the modeling point of view, it turned out that modeling platelets falls apart into platelet adhesion and platelet signaling, each requiring different approaches. As a result, there are mainly three lines of computer model development in the field: models of platelet adhesion, models of platelet signaling, and models of blood coagulation biochemistry. Although there are currently only initial attempts to combine all these aspects of hemostasis in a single model, there are already computer models of almost all elements by themselves.

\section{(C) 2020 Georg Thieme Verlag KG} Stuttgart · New York received

April 14, 2020

accepted after revision

July 6,2020

J) 2020 


\section{A. Platelet adhesion}
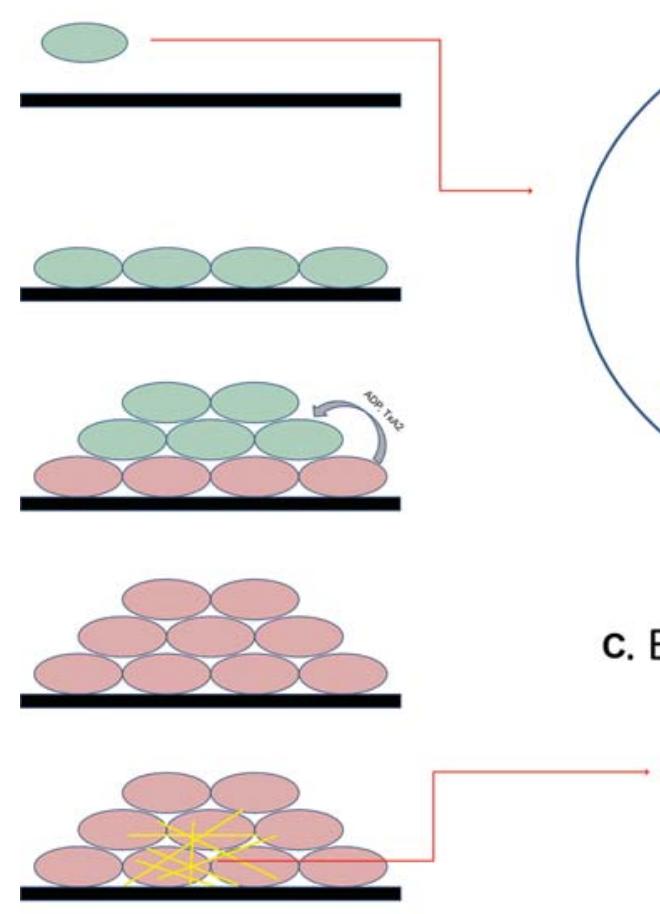

\section{B. Platelet signalling}

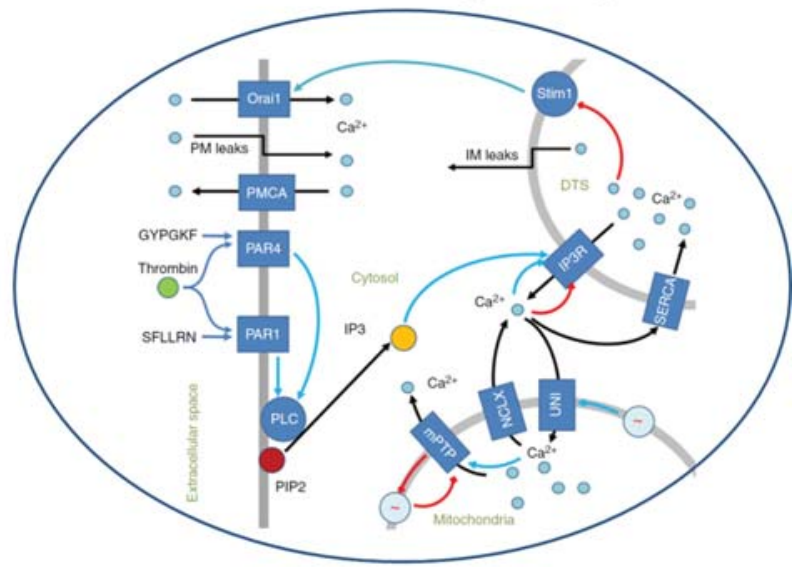

c. Blood coagulation

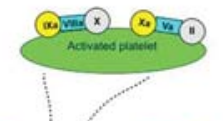

Fig. 1 Elements of the hemostatic response. (A) Platelet adhesion to the site of damage, activation (shown by green to red transition), secretion of ADP and thromboxane A2, and thrombus growth. (B) Some of the platelet signal transduction pathways (the ones activated by thrombin via PAR1 and PAR4 receptors in human platelet ${ }^{55}$ ) that determine the functional response. (C) Blood coagulation network that functions within the thrombus to solidify it. ${ }^{5}$ It is a cascade of enzymatic reactions, with numerous positive and negative feedbacks. It is initiated by binding of the extravascular protein TF to an activated factor VIla and converting it into an active protease enzyme complex. Many reactions of the cascade take place on the activated platelet surface and could be a subject of complicated mathematical models by themselves. ${ }^{11-17}$ The last step is conversion of fibrinogen into fibrin to form the gelatinous clot. PAR, protease-activated receptor; TF, tissue factor.

From the physical point of view, initial platelet plug formation is the formation of a barrier by means of cell aggregation, which is controlled by blood flow and modulation of intercellular binding forces. While flow brings new cells or detaches parts of the aggregate, the modulation of binding is controlled by integrin activation. In addition to platelets, a critical role in this is played by red blood cells (RBCs) that can contribute to both platelet margination in vessels and diffusion. Because of this, the computer models aimed at primary hemostasis or thrombosis are usually "physically challenging," as they require considerations of flowing blood rheology, and cellcell interaction mechanics. The majority of the existing in silico studies therefore focus on solving the physical and computational aspects of platelet adhesion, although some of them attempt to include elements of platelet signal transduction and coagulation pathway. We analyze progress and complications in this field in the first part of this review.

The response of platelets to stimulation is controlled by a complex network of signal transduction (only partially shown in - Fig. 1B) that rapidly integrates signals from numerous receptors (activation by adenosine diphosphate [ADP], colla- gen, thrombin, thromboxane A2, adrenaline, etc.; inhibition by NO and prostacyclin) and forms the ultimate functional responses including adhesion and aggregation (caused by the change in the functional state of integrins), secretion of $\alpha$ - and dense granules, shape change, procoagulant activity, vesiculation, and contraction. Development of the mechanism-driven models of these processes is not usually computationally complex, in contrast to those of platelet transport and adhesion, but requires significant investments into understanding the molecular events that drive intracellular signaling. Analysis of the platelet signal transduction models constitutes the second section of this review.

Finally, blood coagulation ( - Fig. 1C) that goes side by side with fibrinolysis represents a combination of biochemical challenges with complexities of diffusional and convectional transport. ${ }^{10}$ The models that focus on blood coagulation and fibrinolysis biochemistry will be discussed in the third section of this article. Actually, some of the membrane-dependent reactions of blood coagulations are so complex by themselves that large models are designed to deal with their regulation alone ${ }^{11-17}$; the same is true for fibrin polymerization. ${ }^{18}$ 
However, the focus of the present article will be on the models of coagulation as a whole.

The last thing to discuss before going to the models is the goal of the model development: this should definitely affect which models to develop and to what degree. As a matter of fact, the goals of model development is rarely discussed in the field of mathematical and computational biology, often leading experimental researchers to believe that modeling is just an idle exercise. Even now, in the age of computer systems biology, the ideas behind model development are foreign to many experienced hematologists. On more than one occasion, the authors of this review heard from the leading researchers in the hemostatic field questions and statements: "How can you ever be certain that your model is correct?," "Real cell biochemistry is too complex to be represented in a computer model," "I will never believe into these simulations, you can make a sufficiently complex model behave in any manner of your liking" and such. Physical models like the ones discussed in the previous section are more rarely questioned like this, in contrast to the biochemical ones, probably because mechanical modeling is more intuitively obvious. Interestingly, experts in physics and mathematics often do not like models of biochemical networks either, and ask similar questions: the huge biochemical models are too different from the ones traditionally studied in physics and mathematics.

The view of the authors of this article is that a computer model is not different from an experimental model: it is a simplified system that grasps some essential features of the real system sufficiently faithfully so that it could be used to test some hypotheses and generate predictions. It is exactly in the same manner that animal thrombosis models are used for studies before going on to humans. This does not mean that mice no matter how much we humanize them can ever hope to exactly reproduce real-life human clinical conditions; as a matter of fact, in most cases we do not need go too far to make animals human-like, unless we have a reason to believe that we need this. The computer model of a biological network, likewise, is a tool for hypothesis testing and experimental planning. If we believe that something occurs in such-and-such manner (or probably if we have several alternative hypotheses), we can design a model to check whether our ideas of the system structure are in agreement with its behavior. If it is, we can further simulate predictions and test them experimentally to go beyond, and to discover the limits of the model and generate new knowledge.

\section{In Silico Modeling of Arterial Thrombus Formation}

It is generally accepted that primary events of hemostasis and arterial thrombosis involve formation of a platelet aggregate, which is further stabilized by fibrin network. Generally, formation of an aggregate in a wound is called hemostasis. However, some of the vessel-wall injuries result in formation of the massive thrombus in the vessel itself which may lead to vessel occlusion. Such scenario is reproduced in a vast array of in vivo and in vitro models. Importantly, the reason why some of the vessel-wall injuries result in pathological thrombus formation is poorly understood. Thus, the general mechanisms that govern thrombus dynamics and mechanisms of occlusion during both normal and pathological responses to the injury have been a subject of research for several decades.

According to the widely accepted view on arterial thrombus formation, this process involves several critical phenomena, which include adhesion of platelets to the injured vessel wall, as well as to the surface of a growing platelet aggregate, platelet activation, which basically involves integrin activation, shape change, granule release, contraction and formation of procoagulant platelets in some cases, platelet aggregation, which strongly depends on integrin activation, and generation of thrombin, which is a potent activator of platelets, through a coagulation cascade leading to the formation of a fibrin network which is important for stabilizing the aggregate against the flow or against the blood pressure in case if the flow is blocked. Most of the published models are focused on either a particular aspect of the phenomenon or on the general dynamics of thrombus under given conditions.

One of the critical steps for the primary adhesion of platelets is their transport to the injury site which is largely dependent on the platelet margination effect due to plateletRBC interaction at the shear flow. ${ }^{19}$ The mechanisms of platelet margination have been extensively studied using theoretical models based on either macroscopic continuous descriptions ${ }^{20}$ or more detailed simulations resolving single cells. ${ }^{21}$ It has been recently demonstrated in silico that besides standard shear diffusion, platelets could be also involved in a fast transverse transport in the direction to the vessel wall due to transient entrapping in the cavities between bigger RBCs. ${ }^{22}$ Interestingly, such fast transport might be responsible for the rapid margination dynamics which is expected to happen in vivo due to the fact that platelet margination is observed in a circulation system of living animals-where multiple vessel bifurcations and vein valves tend to constantly disturb platelet distribution through the vessel cross-section. Platelet-RBC interactions have been shown to be crucial for the flux of platelets to the vessel wall in the case of a plane surface of in vitro flow chambers and several models of the process have been proposed. ${ }^{23}$

The redistribution of RBCs and platelets in the case of disturbed flow (for example provided by vessel stenosis) was also suggested to influence the primary kinetics of platelet adhesion. ${ }^{24}$ Continuous models describing platelet adhesion using the RBC-platelet interaction-induced flux of the platelet "substance" to the vessel wall or to the surface of platelet aggregates formed as a result of such a flux have been used to study the dynamics of occlusive thrombus formation in the

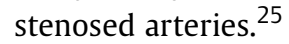

It is now clear that the first steps of platelet-dependent response are driven by purely mechanical processes of the interaction of platelet surface receptors (GPIbalpha and integrins) with their ligands on the surface of the injured vessel wall (von Willebrand factor [vWF], fibrinogen, fibronectin, laminins, etc.). Under conditions of arterial blood flow it is widely accepted that vWF-GPIbalpha interaction is of major importance for primary platelet adhesion. In silico 
models of the process revealed the importance of both the discoid platelet shape ${ }^{26}$ and vWF length for efficient adhesion. $^{27}$

Formation of the platelet aggregate in the case of disturbed flow described in various experimental models ${ }^{28,29}$ seems to represent another biomechanical phenomenon which strongly depends on the vWF-GPIbalpha interaction. It has been demonstrated that activation of A1 domain interaction of vWF with GPIbalpha follows the flow-induced unfolding of the multimers, ${ }^{30,31}$ which might happen both on the surface and in the bulk solution. However, the detailed physical description of the processes leading to platelet aggregation under disturbed flow is still missing. One of the suggested mechanisms involves vWF unfolding ${ }^{32}$ due to possible presence of significant elongational velocity gradients in case of stenosis. Interestingly, this hypothesis implies that the vWF activation process should occur in the bulk at some distance from the surface. Such a possibility has been recently analyzed using the coarse-grained model of vWF dynamics under conditions corresponding to an in vitro model of vessel stenosis. ${ }^{29}$

During the last decades, dozens of thrombus formation models were developed addressing various knowledge gaps in this complex phenomenon. One of the intriguing questions regarding the mechanism triggering the occlusion has been addressed by the simplified model of thrombus growth under constant pressure boundary conditions. ${ }^{33}$ Using this model, it was demonstrated that the size of the injury might represent the crucial parameter, which determines the thrombus fate due to dependence of the maximum shear rate generated on thrombus surface on the hydraulic resistance of the thrombus. In this model, the total flux of the platelets to thrombus surface was considered to depend solely on the surface shear rate, which was shown to be a function of both thrombus height and length due to constant pressure boundary conditions.

The most recent in silico models of thrombus formation are focused on the origin and the consequences of internal thrombus heterogeneity. It was shown for both microvasculature and macrovasculature that laser-induced injuries generate nonocclusive mural thrombi possessing two-stage dynamics (growth followed by shrinkage) and pronounced internal heterogeneity (having a "core" of highly activated platelets close to the injury size and a mobile "shell" of reversibly activated discoid platelets which covers the "core").34,35 This "core and shell" model of the hemostatic thrombus received much attention in the field, so here we aimed at formulation of some questions that we believe are important and that might be tackled using novel in silico models ( - Fig. 2), either particle-based ${ }^{36,37}$ or continuous. ${ }^{38}$

The origin of such heterogeneity has been a subject of several computational studies, ${ }^{39,40}$ which were focused on the impact of agonist transport parameters within thrombus on the local platelet density and corresponding geometry of interplatelet space. Using the continuous viscoelastic model of the preformed heterogeneous clots, it was recently shown that the stability of such thrombi against the flow is largely dependent on the internal permeability distribution. ${ }^{41}$
Another continuous model of thrombus formation which takes into account platelet aggregation, secretion, and coagulation has been used to study the dynamics of thrombus formation on the tissue factor (TF) observed in vitro under venous shear rates. This model predicted the dependence of both clot size and internal composition on the surface density of the TF and the length of the corresponding region. ${ }^{42}$

They key mechanism responsible for internal thrombus heterogeneity is contraction. Contraction is a process which is driven by mechanical coupling of actomyosin complexes inside platelet and integrins on the surface of platelet. This active ATP-dependent process leads to overall contraction of the platelet aggregate due to internal forces acting within each platelet which are translated to interplatelet contacts through fibrinogen bridges between integrins. This process has been described in both in vitro and in vivo arterial thrombi and was recently demonstrated to be the driving mechanism of procoagulant platelet redistribution within the growing thrombus. ${ }^{43}$ To describe the mechanics of the process, a simple particle-based model of platelet aggregate contraction based on Morse potential has been introduced. ${ }^{43}$

The particle-based model with platelet-platelet interaction described using Morse potential was recently developed and utilized to describe platelet aggregation observed under normal and disturbed flow conditions. ${ }^{36}$ Another particlebased model of thrombus dynamics with quasi-steady flow approximation and heterogeneous particle sizes was shown ${ }^{44}$ to successfully reproduce the parameters of shell dynamics observed in vivo.

The dynamics of the outer layers of heterogeneous thrombus was suggested to play an important role in the regulation of thrombus size and was studied using the dissipative particle dynamics model. ${ }^{37,45}$ After the removal of the outer thrombus layers, the surface of the remaining fibrin-rich thrombus was suggested to be weakly adhesive, thus preventing further thrombus propagation and suggesting a mechanism for regulation of thrombus size. Another mechanism, which was suggested to limit the propagation of thrombus, was studied in a continuous model of thrombosis ${ }^{46}$ and emphasized the role of the hindered transport of species in the internal regions of the thrombus leading to the decrease of substrate supply and hence lowering of the thrombin production rate.

To conclude, we would like to emphasize that despite several interesting mechanisms of thrombus size regulation were suggested and studied in silico during the last decade, the factors determining the observed dynamics of nonocclusive thrombi in vivo as well as the mechanisms responsible for triggering occlusive thrombosis are still not clear. The effects of conditions, such as the vessel size or geometry of the damaged area or biochemistry of the vessel wall, are still poorly understood.

\section{Computer Models of Platelet Signal Transduction and Function}

Following the overall timeline of signal transduction research, in silico modeling of platelet function has been lagging behind. Important predictions from computer models in blood 
A

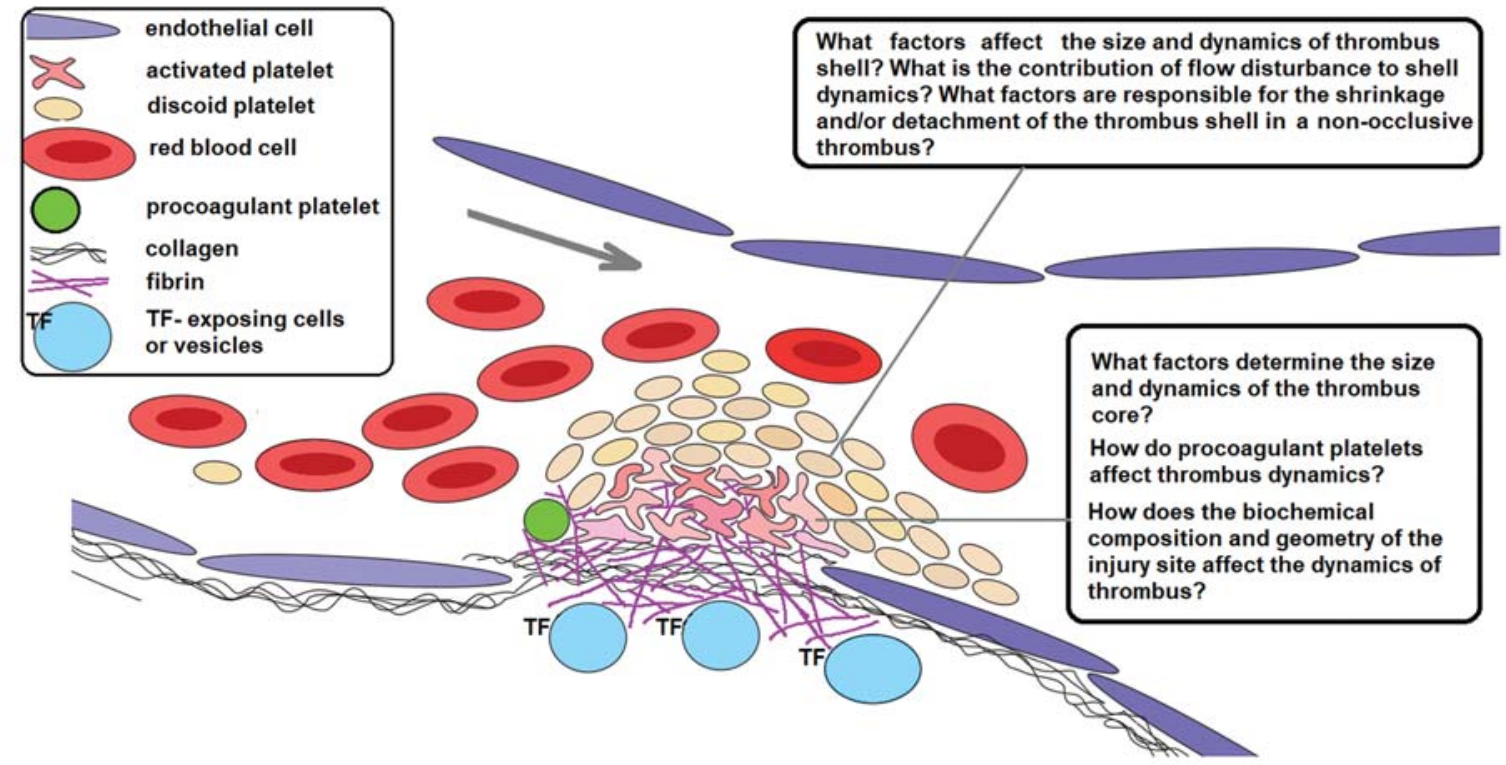

B

(a)

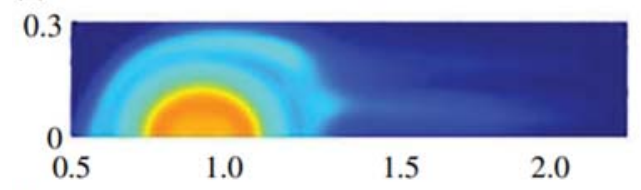

(b)

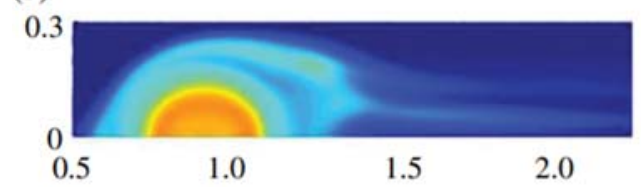

(c)
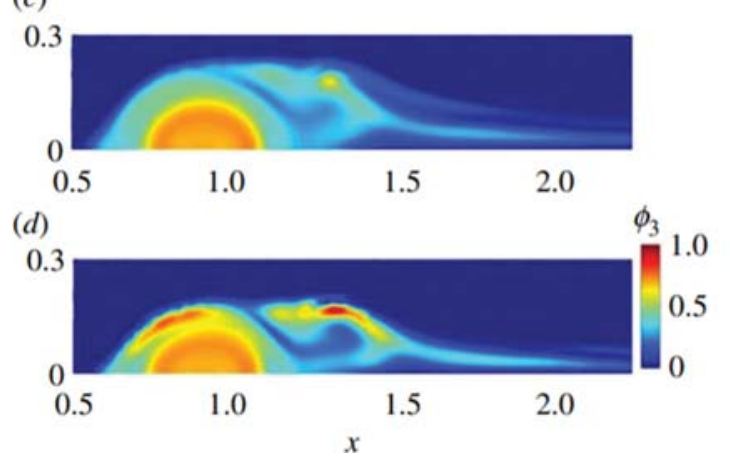

C
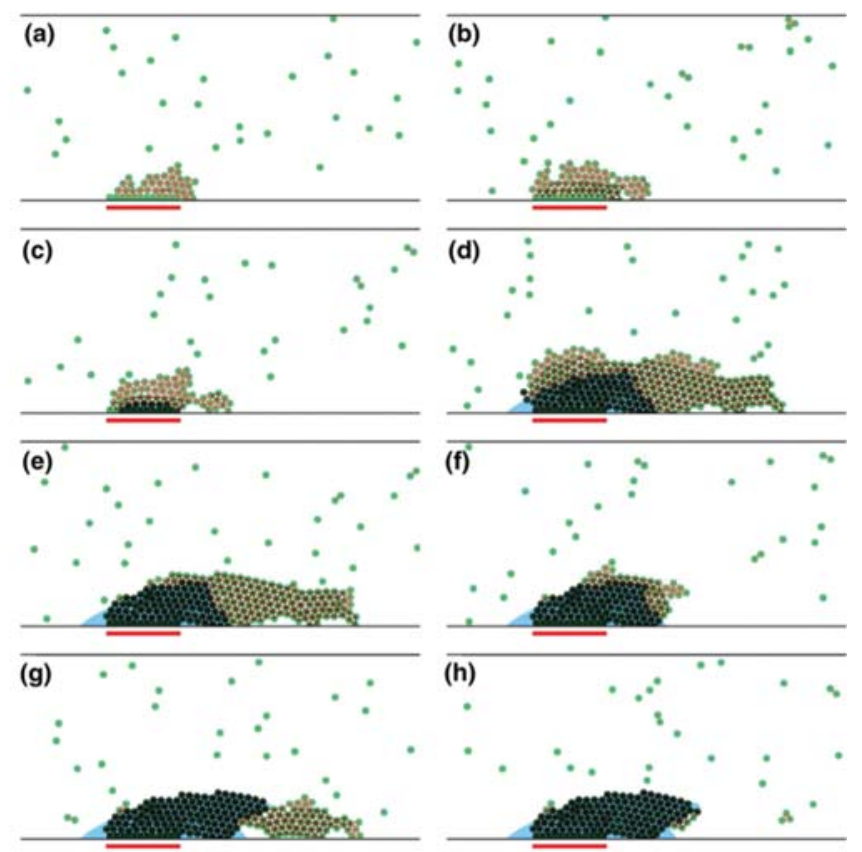

Fig. 2 Schematic illustration of the current views and questions, as well as model examples. (A) "Core and shell” concept of arterial thrombus structure and corresponding questions. (B) An example of the results obtained using a continuous model of microvascular thrombus, where blood clot stability and clot fraction were analyzed at an increasing flow shear rate for heterogeneous thrombi. ${ }^{41}$ (C) Example of thrombus dynamics obtained using the dissipative particle dynamics model of thrombus formation. ${ }^{45}$ The detachment of the outer layer of thrombus (with no fibrin) is demonstrated.

coagulation appeared as early as $1989,{ }^{47}$ while first thrombus formation models with sufficiently complicated mechanisms of platelet adhesion were designed a decade later, by $1999 .{ }^{48}$ For comparison, a pioneering study to describe the signal transduction pathway for platelet stimulation by ADP via P2Y1 receptor leading to calcium increase appeared only one more decade later, in $2008 .{ }^{49}$ This was followed by a sharp rise in the number of models that focused on different signal transduction pathways (see a comprehensive recent list in, e.g., Dunster et $\mathrm{al}^{3}$ ) and even traced some of the pathways down to the functional responses, like phosphatidylserine exposure. ${ }^{50}$ Models of platelet metabolism were also proposed, ${ }^{51}$ as well as combinations of signal transduction with metabolic regulation. ${ }^{52}$ Here we shall neither go into the history of the development of these models, nor design an updated comprehensive list of them, nor dive into the methodology (more specialized papers like that of Dunster et $\mathrm{al}^{3}$ are more suitable for this), but rather focus on the main questions: what they are, how do the models of signal 
transduction fit into development of in silico models of hemostasis and thrombosis, what do we need to make them fulfill this role better, and what are the other purposes of their application.

The computer models of platelet signal transduction and function are usually sets of ordinary differential equations (ODEs) that are based on the laws of chemical kinetics, where variables are concentrations of species in the cytoplasm or other compartments. For example, the first step to describe P2Y12 activation by $\mathrm{ADP}^{53}$ is to calculate the rate of their binding and dissociation determined by the law of mass action for their concentrations:

$$
V=k[\mathrm{P} 2 \mathrm{Y} 12] \cdot[\mathrm{ADP}]-k_{\mathrm{m}} \cdot[\mathrm{P} 2 \mathrm{Y} 12-\mathrm{ADP}](1)
$$

The platelet shape and volume change are usually not considered in these models, so they do not have "mechanical" parts. The small size of platelets usually does not warrant consideration of transport processes either, so use of partial differential equations in their models of signal transduction (which could be the case with larger cells) is not usually necessary. There could be exceptions if nonuniform membrane processes like lipid raft involvement are considered (see the recent example of CLEC-2 signal transduction model in platelets ${ }^{54}$ ). In contrast, stochastic algorithms might be needed because some of the species could be at low quantities (less than 100-1,000 molecules), which makes the use of ODE inappropriate. ${ }^{50,55}$ As a result, the models of platelet signal transduction are usually relatively simple computationally and can be solved using various commercial or freeware ODE solvers, or specialized biochemical simulators like COPASI. ${ }^{56}$ In contrast, the main challenge is usually biochemical: the signaling network is large and poorly characterized, so its modeling requires in-depth analysis and a lot of information. Recent progresses on platelet quantitative proteomics, ${ }^{57,58}$ as well as new methods of signaling monitoring, make development and validation of such models much more accessible and reliable, but the remaining challenges are still great.

Going back to platelet signaling, the first way to use the models is exactly to identify issues in our understanding of signaling pathways, to test suggestions about regulation and about the roles of proteins and reactions. The examples of successful models are usually not the stories of model development but rather stories of hypotheses tested and predictions performed; hypotheses that were too complex to be addressed with pure experiments. Roles of the negative feedback in the regulation of platelet activation via GPVI, ${ }^{59}$ importance of spatial receptor distribution rather than copy numbers in the regulation of the response, ${ }^{60}$ identification of the controlling stages in CLEC-2 signaling, ${ }^{54}$ developing hypotheses for heterogeneity of integrin response in immune thrombocytopenia, ${ }^{61}$ the role of mitochondrial calcium integration in procoagulant platelet formation, ${ }^{50,62}$ importance of platelet surface-to-volume ratio and the number of mitochondria in programmed cell death of Wiskott-Aldrich syndrome platelets as a possible mechanism behind thrombocytopenia, ${ }^{52}$ and interplay of protease-activated receptor 1 (PAR1) and PAR4 to form the optimal response of platelets to thrombin in time- and concentration-dependent manner ${ }^{55}$; these are examples of how one might use a model of a signaling pathway to learn new information about the system.

As an example, let us consider a relatively straightforward problem of interplay between PAR1 and P2Y12 receptors in procoagulant platelet formation. It has been long known that although ADP cannot induce phosphatidylserine externalization, it can significantly increase the number of procoagulant platelets produced by thrombin or dual stimulation by providing additional activation via P2Y12 receptor. ${ }^{63}$ To gain insight into this, we designed a model including both of these pathways (-Fig. 3A) and incorporated a potential mechanism of phospholipase C-beta regulation by a catalytic subunit of protein kinase A. The model simulations (-Fig. 3B) showed that this mechanism was sufficient to explain the results of the experiments (-Fig. 3C). However, the model also predicted that even greater effects could be achieved by adding ADP before PAR stimulation, because it takes some time for the cAMP level to fall, and this prediction was confirmed experimentally (-Fig. 3D). This, in turn, made us to propose that this could be important in thrombus formation because it is likely that platelets in the thrombus are first activated by rapidly diffusing ADP and only then by thrombin (-Fig. 3E).

This example shows the logic of using a model to obtain information about a signaling pathway that can then potentially lead to a physiologically meaningful response. Of course, to go further, one would have to integrate signaling models (probably, in a simplified manner) with thrombus formation models. One example of this is a pioneering study of Flamm et $\mathrm{al}^{64}$ where a platelet signaling model represented by a neural network is coupled with a platelet deposition model.

Still, it is vital to underline that it is not necessary to create a super-integrated model to obtain a physiologically meaningful result, to understand a disease, or to test a target candidate. Among the examples cited above, there was a model that predicted that platelets of the patients with Wiskott-Aldrich syndrome will less likely undergo necrosis if they are large ${ }^{64}$; the experiments revealed that indeed the platelet count significantly correlates with the platelet size in the WAS patients. One can use a model to make a step at the level of signal transduction only, and then extend the conclusions to thrombus formation or other purpose using other tools.

\section{Computer Models of Blood Coagulation and Fibrinolysis}

Blood coagulation cascade, a complex network of biochemical reactions leading to fibrin formation, became one of the first biological systems whose outcome was described in mathematical terms starting at least from $1966,{ }^{65}$ and since then it has been implemented in some way in numerous papers. These studies, which initially were mostly aimed at research, have been recently increasingly used for biomedical developments: the examples over the last year include clustering of thrombin generation diagnostic data, ${ }^{66}$ investigation of thrombin inhibitors, ${ }^{67}$ phenotype discrimination in hemophilia, ${ }^{68}$ mechanisms of impaired coagulation in neonates, ${ }^{69}$ and dilutional coagulopathy. ${ }^{70}$ 


\section{A. The network}

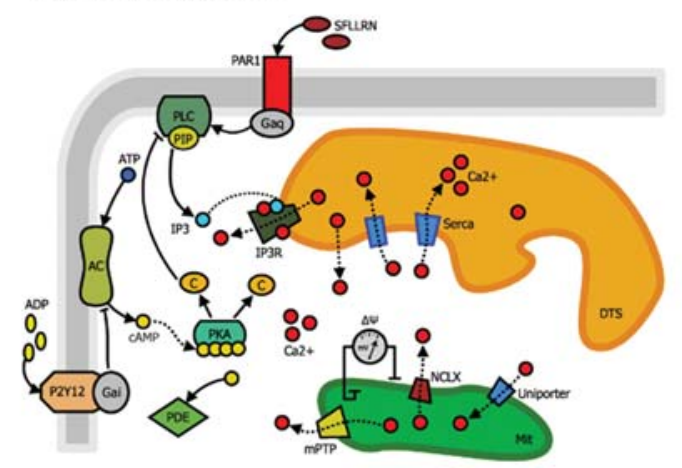

B. The model

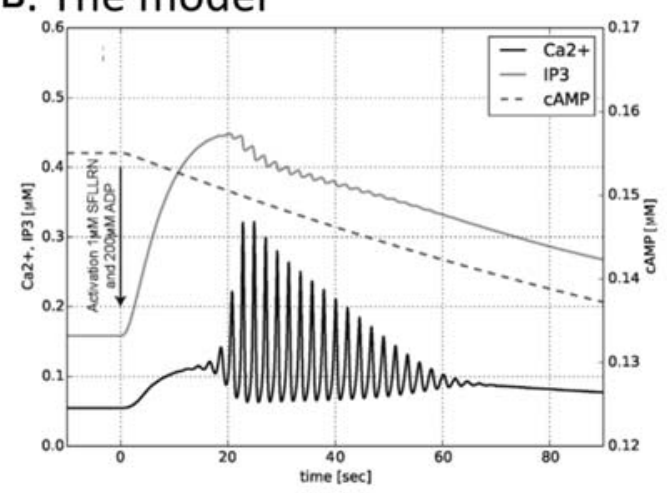

c. Validation

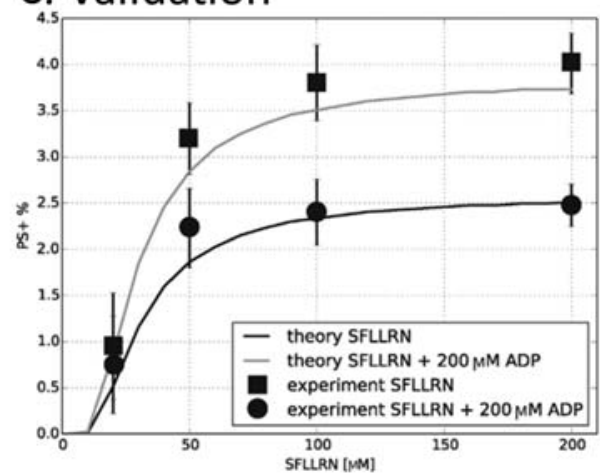

D. Prediction

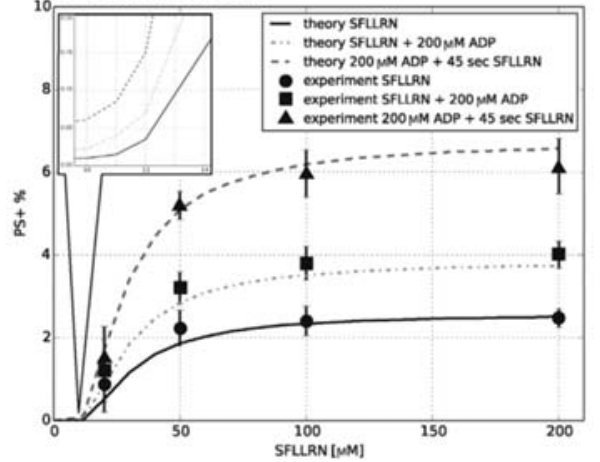

E. Potential significance

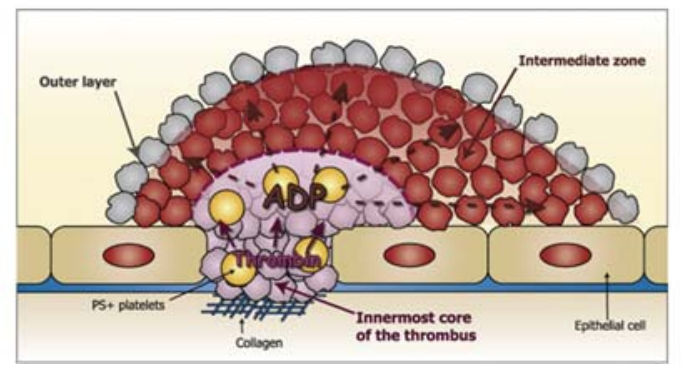

Fig. 3 Interplay of PAR1 and P2Y12: insights from a mathematical model. ${ }^{53}$ (A) The main reactions of the model. The system includes two activators, thrombin receptor activation peptide SFLLRN that initiates calcium oscillation by acting via Gq-coupled receptor PAR1, and ADP that decreases cAMP by acting on its Gi-coupled receptor P2Y12. The pathways converge at the level of PLC that depends on PKA. (B) Dynamics of the essential variables: SFLLRN raises IP3 and induces calcium oscillations, while ADP decreases CAMP with relatively slow characteristic times. (C) Procoagulant platelet formation as a function of thrombin-activating peptide (SFLLRN) and ADP concentration when added together. (D) Procoagulant platelet formation as a function of thrombin-activating peptide (SFLLRN) and ADP concentration when added one after another: when ADP has some time to act, it can decrease cAMP greater and increase platelet sensitivity to SFLLRN. (E) Potential role of the ADP-dependent preactivation in thrombus formation, as a preparation for thrombin stimulation: rapidly diffusing and easily released ADP precedes thrombin during thrombus formation and sensitizes the platelets on its way in a concentration-dependent manner. cAMP, cyclic adenosine monophosphate; PAR, protease-activated receptor; PLC, phospholipase C; PKA, protein kinase A.

There are several reasons why this system was so widely used as an object of modeling. First, it is pretty well investigated, and a lot is known about its participants: concentrations and reactions with their rates. Second, it is complex, and simple logical conclusions on the impact of one or another parameter are difficult without mathematical analysis. Third, it is relevant for human health, thus it has value aside pure math. Here we will describe the basic principles and current state in mathematical simulations of blood coagulation and fibrinolysis.

The models of blood coagulation and fibrinolysis are, similarly to the models of platelet signaling, sets of differential equations based on the laws of chemical kinetics. Stochastic simulations are used very rarely. ${ }^{71}$ However, in contrast to platelet signaling, spatial heterogeneity is vital, so quite several of these models consider spatial propagation of the fibrin clot or its dissolution, ${ }^{72-79}$ while others could include this implicitly. ${ }^{80}$

Models of blood coagulation (and any biological system as well) can be divided in two groups based on the principle of how they describe the object of simulation. These groups are phenomenological and mechanistic models. The first describes the system using some general rules; for blood coagulation, they may be like these: the clot must be solid; 
the transition from the liquid to the solid state must occur fast; the clot must be localized near the damaged site and it must have a definite border with liquid blood. Based on such assumptions, a model of two partial differential equations was developed, ${ }^{81}$ which described a self-sustaining wave of clotting propagation, later found experimentally. ${ }^{73}$ Such types of models are suitable for hypothesis testing, investigation of some basic features of the system. The second type of models, mechanistic, describes the whole system or part of it using experimentally observed reactions, with measured reaction rates. ${ }^{82}$ These models can be used for detailed investigation of the system's properties, regulations at different scales, and interaction with therapeutic agents.

Although the phenomenological models of blood coagulation are not very popular nowadays, when computational power is sufficient for simulation of almost any model we can propose, these simple models played a noticeable role in the understanding of mechanisms of clotting regulation and, in our opinion, they are still a powerful instrument, which should not be neglected. In Beltrami and Jesty, ${ }^{83}$ analysis of positive feedback loops in regard to response in systems with activation thresholds was performed. The authors found that a long-range positive feedback (when final enzyme produced in the last loop activated the initial step in the first loop) was able to overcome the threshold in the case of a high catalytic rate, or to make the system stable, i.e., abolish the oscillatory behavior. Later, the importance of a long-range feedback of thrombin-catalyzed factor XI activation for blood coagulation propagation was shown experimentally. ${ }^{73}$ A system with a positive feedback loop localized on a membrane in contact with a flowing medium was examined by Beltrami and Jesty, ${ }^{84}$ and they found that the feedback threshold was controlled by the flow rate of the adjacent medium and the physical size of the membrane patch. In terms of blood coagulation, it means that blood clotting onset depends on the blood flow velocity and the size of the damaged area in a threshold manner. This finding was experimentally confirmed. ${ }^{85}$

A series of mechanism-driven models originating from the work of Panteleev et al, ${ }^{75}$ which included a comprehensive description of the coagulation network, coagulation factor interactions with platelets, and transport processes, was aimed at the regulation of blood coagulation in space and time. - Fig. 4 shows an early example of the use of a model to study the role of intrinsic tenase in fibrin clot growth. Experimental size of the fibrin clot versus time ( - Fig. $4 \mathrm{~A}$ ) is reproduced in a validated model ( $\sim$ Fig. $4 B$ ), which allows us to gain insight into the contribution of intrinsic and extrinsic tenases to factor Xa generation in space and time ( - Fig. 4C): one can notice that extrinsic tenase works near the activator, while extrinsic tenase is the main producer far from it.

These studies investigated the mechanisms behind thrombus growth and its stopping, ${ }^{73,75}$ the role of flow in the regulation of coagulation pathways, ${ }^{77}$ formation of the
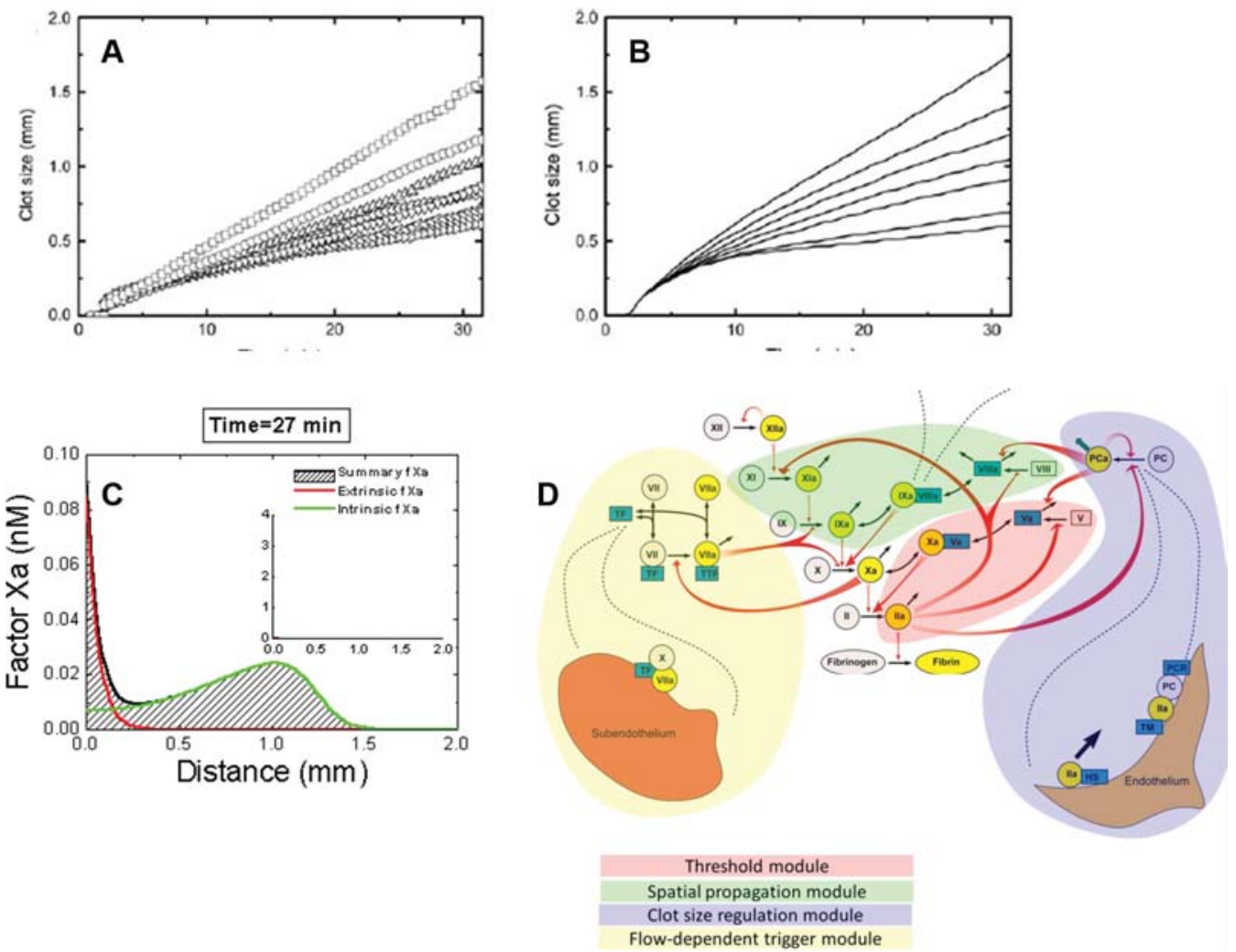

Fig. 4 Spatial regulation of coagulation: insights from a mathematical model. ${ }^{75}$ (A) Fibrin clot size as a function of time for different concentrations of factor VIII: the experiment. (B) The same for computer simulations. Notice that factor VII does not affect lag-time in either model or experiments, but rather regulates the spatial velocity. (C) Use of the model to identify contributions of extrinsic and intrinsic tenase to factor Xa formation near and far from the activator. (D) Modular decomposition of blood coagulation, where parts of the system responsible for different aspects of its behavior are shown. ${ }^{86}$ 
activation threshold in the clotting system, ${ }^{86}$ importance of spatial TF distribution ${ }^{72}$ and regulatory roles of TF pathway inhibitor, ${ }^{76}$ drug-drug interaction in hemophilia, ${ }^{87}$ and others. Recently, a detailed model of spatial thrombin generation and clot formation consisting of 21 partial differential equations and three ODEs was used to investigate blood coagulation sensitivity to coagulation factor deficiencies. ${ }^{88}$ The authors concluded that the spatially heterogeneous coagulation system was robust to factor concentration variation due to combination of local high TF surface, diffusion control being shared between different active factors, and early saturated dependence of fibrin clot formation by thrombin. These studies ultimately led to the proposal of a modular concept of blood coagulation network ( - Fig. 4D).

If phenomenological models can supply us with qualitative behavior of the clotting system, mechanistic models are often aimed to produce quantitative information. The main protein of clotting is thrombin, which not only activates fibrinogen and produces clot, but also regulates most of the reactions of blood coagulation and activates platelets. In vitro thrombin generation assay provides time course of thrombin concentration in the examined sample during clotting, and this experimental setup is easy for computer simulations. One of the first mathematical models simulating the whole system was the model of Kenneth Mann's group, ${ }^{82}$ which used 34 ODEs to describe thrombin generation by interaction of TF, VII, VIIa, X, IX, II, VIII, V, TF pathway inhibitor, and antithrombin. This model is still used as a basis for more complex integrated models of thrombin generation, fibrin formation, and fibrinolysis, also containing several reactions that are specific to the thrombin generation assay and can affect the thrombin readout. ${ }^{89,90}$ Such an approach was used to simulate effects of plasma dilution, hypothermia, and acidosis. ${ }^{70}$ They found that dilution progressively reduced the peak of thrombin generation; interestingly, in an experimental report, moderate hemodilution increased thrombin generation. ${ }^{91}$ In these simulations hypothermia delayed the onset of thrombin generation and increased thrombin generation (both endogenous thrombin potential and thrombin peak). Another group used the legacy model to describe how changes in blood coagulation factor balance in human immunodeficiency virus patients shift the hemostasis to hypercoagulation, ${ }^{92}$ or in patients with hemophilia $C,{ }^{93}$ hemophilia $\mathrm{A},{ }^{94}$ or deep vein thrombosis, ${ }^{95}$ and what is the variation of thrombin generation in healthy individuals. ${ }^{96}$ Although the computer simulations of thrombin generation often demonstrate the same trends in response to parameters' variations as in vitro experiments, the thorough comparison of the outputs can show that the discrepancy in the in vitro and in silico is still present, ${ }^{97}$ and it is as high as 30 to $50 \%$ of values of thrombin peak, or time to peak. It should be noted that the thrombin generation assay itself has great variability, ${ }^{98}$ and results from different laboratories can vary up to $20 \%,{ }^{99}$ but even the position of the sample in the microplate can change the output up to $30 \% .{ }^{100}$

Aside of blood coagulation, the fibrin clot lysis process is less extensively studied and simulated. Fibrinolysis is an endpoint stage of clot existence, and its behavior depends on the clot- forming process: the structure of the fibrin clot, defined during clot formation, regulates the rate of clot dissolution. Simple models of fibrinolysis were implemented for investigation of different aspects of clot dissolution. Models of diffusion and permeation process during clot lysis demonstrated that pressure-driven permeation is the major mode of transport that allows for kinetically significant thrombolysis during clinical situations. ${ }^{74} \mathrm{~A}$ three-dimensional stochastic multiscale model of fibrinolysis with a single-fiber cross-section at the microscale and a three-dimensional (3D) fibrin clot at the macroscale ${ }^{101}$ demonstrated that number of tissue plasminogen activator (tPA) molecules relative to the surface area of the clot regulated the rate of clot dissolution, explaining the mechanism by which coarse clots lysed more quickly than fine clots. The same model was used to investigate the lysis propagation regime. ${ }^{102}$ The authors found that the physical mechanism of plasmin action was crawling across a fiber using neighbor binding sites, and it coupled with plasmin-regulated local concentration of tPA defined the kinetic conditions necessary for fibrinolysis to proceed as a front.

A combination of a spatially distributed model of clot formation from Kuprash et $\mathrm{al}^{88}$ and a model of clot lysis resulted in a set of 32 partial differential equations and three ordinary differential equations. ${ }^{78}$ The authors investigated mechanisms regulating simultaneous clot formation and dissolution. They found that lysis front propagation followed front of clot formation with velocity linearly proportional to the velocity of clotting front, as fibrin formation was the ratelimiting reaction.

Besides finding of mechanisms of blood coagulation and fibrinolysis regulation, mathematical modeling is widely used for the evaluation of drug action, dose-dependent safety and efficacy, and pharmacodynamic profiles. In the work of Shibeko et $\mathrm{al}^{103}$ using a model of factor Xa generation via TFdependent and lipid-dependent pathways and a biphasic pharmacokinetic model of factor VIIa (FVIIa), different modifications of FVIIa variants were investigated and it was shown that some potent modifications of the rFVIIa molecule may not translate into a prolonged hemostatic effect.

Simulation of fibrinolysis in a 3D patient-specific artery during thrombolytic therapy showed that clot lysis was accelerated at higher tPA doses at the expense of a substantial increase in the risk of intracerebral hemorrhage, which was supposed to be associated with fibrinogen consumption. ${ }^{104}$

Mathematical modeling in blood coagulation is a potent tool for investigation of mechanisms regulating different aspects of clotting, such as thrombin generation, clot dissolution, action of different drugs, used for hypo- and hypercoagulation correction, and for thrombolysis. Yet, the direct translation of in silico results to in vitro/ex vivo setups is apparently too premature and requires further investigations of clotting regulating parameters.

\section{Conclusions}

- During the last decade, different groups have developed several approaches to simulate all critical components of 
the hemostatic response including platelet adhesion, platelet signal transduction, and blood coagulation.

- Even by themselves, these models are being used for experimental planning and prediction, to get insights into the regulation of hemostasis and thrombosis, identify roles of proteins and reactions, select drug targets, and optimize diagnostic assays that are simpler to mimic real hemostasis.

- From the technical point of view, these models are being constantly improved to include more and more essential elements, corrected mechanisms, and better ways of simulation. They are already being combined to provide descriptions of in vivo hemostasis and thrombosis at different scales.

\section{Conflict of Interest}

The authors declare, that they have no conflict of interest.

\section{Acknowledgments}

The authors were supported by a Russian Science Foundation grant (17-74-20045).

\section{References}

1 Xu Z, Kamocka M, Alber M, Rosen ED. Computational approaches to studying thrombus development. Arterioscler Thromb Vasc Biol 2011;31(03):500-505

2 Shibeko AM, Panteleev MA. Untangling the complexity of blood coagulation network: use of computational modelling in pharmacology and diagnostics. Brief Bioinform 2016;17(03): 429-439

3 Dunster JL, Panteleev MA, Gibbins JM, Sveshnikova AN. Mathematical techniques for understanding platelet regulation and the development of new pharmacological approaches. Methods Mol Biol 2018;1812:255-279

4 Belyaev AV, Dunster JL, Gibbins JM, Panteleev MA, Volpert V. Modeling thrombosis in silico: frontiers, challenges, unresolved problems and milestones. Phys Life Rev 2018;26-27:57-95

5 Panteleev MA, Andreeva AA, Lobanov AI. Differential drug target selection in blood coagulation: what can we get from computational systems biology models? Curr Pharm Des 2020;26(18): 2109-2115

6 Panteleev MA, Ananyeva NM, Ataullakhanov FI, Saenko EL. Mathematical models of blood coagulation and platelet adhesion: clinical applications. Curr Pharm Des 2007;13(14): 1457-1467

7 Ataullakhanov FI, Panteleev MA. Mathematical modeling and computer simulation in blood coagulation. Pathophysiol Haemost Thromb 2005;34(2-3):60-70

8 Nesbitt WS, Mangin P, Salem HH, Jackson SP. The impact of blood rheology on the molecular and cellular events underlying arterial thrombosis. J Mol Med (Berl) 2006;84(12):989-995

9 Swieringa F, Spronk HMH, Heemskerk JWM, van der Meijden PEJ. Integrating platelet and coagulation activation in fibrin clot formation. Res Pract Thromb Haemost 2018;2(03):450-460

10 Panteleev MA, Dashkevich NM, Ataullakhanov FI. Hemostasis and thrombosis beyond biochemistry: roles of geometry, flow and diffusion. Thromb Res 2015;136(04):699-711

11 Kovalenko TA, Panteleev MA, Sveshnikova AN. Substrate delivery mechanism and the role of membrane curvature in factor $\mathrm{X}$ activation by extrinsic tenase. J Theor Biol 2017;435:125-133

12 Panteleev MA, Saenko EL, Ananyeva NM, Ataullakhanov FI. Kinetics of factor $X$ activation by the membrane-bound complex of factor IXa and factor VIIIa. Biochem J 2004;381(Pt 3):779-794
13 Panteleev MA, Zarnitsina VI, Ataullakhanov FI. Tissue factor pathway inhibitor: a possible mechanism of action. Eur J Biochem 2002;269(08):2016-2031

14 Podoplelova NA, Sveshnikova AN, Kotova YN, et al. . Coagulation factors bound to procoagulant platelets concentrate in cap structures to promote clotting. Blood 2016;128(13):1745-1755

15 Podoplelova NA, Sveshnikova AN, Kurasawa JH, et al. . Hysteresislike binding of coagulation factors $\mathrm{X} / \mathrm{Xa}$ to procoagulant activated platelets and phospholipids results from multistep association and membrane-dependent multimerization. Biochim Biophys Acta 2016;1858(06):1216-1227

16 Terentyeva VA, Sveshnikova AN, Panteleev MA. Kinetics and mechanisms of surface-dependent coagulation factor XII activation. J Theor Biol 2015;382:235-243

17 Zakharova NV, Artemenko EO, Podoplelova NA, et al. . Platelet surface-associated activation and secretion-mediated inhibition of coagulation factor XII. PLoS One 2015;10(02):e0116665

18 Weisel JW, Nagaswami C. Computer modeling of fibrin polymerization kinetics correlated with electron microscope and turbidity observations: clot structure and assembly are kinetically controlled. Biophys J 1992;63(01):111-128

19 Goldsmith HL, Turitto VT. Rheological aspects of thrombosis and haemostasis: basic principles and applications. ICTH-ReportSubcommittee on Rheology of the International Committee on Thrombosis and Haemostasis. Thromb Haemost 1986;55(03): 415-435

20 Tokarev AA, Butylin AA, Ermakova EA, Shnol EE, Panasenko GP, Ataullakhanov FI. Finite platelet size could be responsible for platelet margination effect. Biophys J 2011;101(08):1835-1843

21 Czaja B, Gutierrez M, Závodszky G, de Kanter D, Hoekstra A, Eniola-Adefeso $\mathrm{O}$. The influence of red blood cell deformability on hematocrit profiles and platelet margination. PLOS Comput Biol 2020;16(03):e1007716

22 Vahidkhah K, Diamond SL, Bagchi P. Platelet dynamics in threedimensional simulation of whole blood. Biophys J 2014;106(11): 2529-2540

23 Tokarev AA, Butylin AA, Ataullakhanov FI. Platelet adhesion from shear blood flow is controlled by near-wall rebounding collisions with erythrocytes. Biophys J 2011;100(04):799-808

24 van Rooij BJM, Závodszky G, Azizi Tarksalooyeh VW, Hoekstra AG. Identifying the start of a platelet aggregate by the shear rate and the cell-depleted layer. J R Soc Interface 2019;16(159): 20190148

25 Bark DL Jr, Ku DN. Platelet transport rates and binding kinetics at high shear over a thrombus. Biophys J 2013;105(02):502-511

26 Mody NA, Lomakin O, Doggett TA, Diacovo TG, King MR. Mechanics of transient platelet adhesion to von Willebrand factor under flow. Biophys J 2005;88(02):1432-1443

27 Belyaev AV. Long ligands reinforce biological adhesion under shear flow. Phys Rev E 2018;97(4-1):042407

28 Nesbitt WS, Westein E, Tovar-Lopez FJ, et al. . A shear gradientdependent platelet aggregation mechanism drives thrombus formation. Nat Med 2009;15(06):665-673

29 Receveur N, Nechipurenko D, Knapp Y, et al. . Shear rate gradients promote a bi-phasic thrombus formation on weak adhesive proteins, such as fibrinogen in a VWF-dependent manner. Haematologica 2019 (e-pub ahead of print) . Doi: 10.3324/ haematol.2019.235754

30 Fu H, Jiang Y, Yang D, Scheiflinger F, Wong WP, Springer TA. Flowinduced elongation of von Willebrand factor precedes tensiondependent activation. Nat Commun 2017;8(01):324

31 Deng W, Wang Y, Druzak SA, et al. . A discontinuous autoinhibitory module masks the A1 domain of von Willebrand factor. J Thromb Haemost 2017;15(09):1867-1877

32 Schneider SW, Nuschele S, Wixforth A, et al. . Shear-induced unfolding triggers adhesion of von Willebrand factor fibers. Proc Natl Acad Sci U S A 2007;104(19):7899-7903 
33 Belyaev AV, Panteleev MA, Ataullakhanov FI. Threshold of microvascular occlusion: injury size defines the thrombosis scenario. Biophys J 2015;109(02):450-456

34 Stalker TJ, Traxler EA, Wu J, et al. . Hierarchical organization in the hemostatic response and its relationship to the plateletsignaling network. Blood 2013;121(10):1875-1885

35 Welsh JD, Poventud-Fuentes I, Sampietro S, Diamond SL, Stalker TJ, Brass LF. Hierarchical organization of the hemostatic response to penetrating injuries in the mouse macrovasculature. J Thromb Haemost 2017;15(03):526-537

36 Yazdani A, Li H, Humphrey JD, Karniadakis GE. A general sheardependent model for thrombus formation. PLOS Comput Biol 2017;13(01):e1005291

37 Tosenberger A, Ataullakhanov F, Bessonov N, Panteleev M, Tokarev A, Volpert V. Modelling of thrombus growth in flow with a DPD-PDE method. J Theor Biol 2013;337:30-41

38 Babushkina ES, Bessonov NM, Ataullakhanov FI, Panteleev MA. Continuous modeling of arterial platelet thrombus formation using a spatial adsorption equation. PLoS One 2015;10(10): e0141068

39 Tomaiuolo M, Stalker TJ, Welsh JD, Diamond SL, Sinno T, Brass LF. A systems approach to hemostasis: 2. Computational analysis of molecular transport in the thrombus microenvironment. Blood 2014;124(11):1816-1823

40 Mirramezani M, Herbig BA, Stalker TJ, et al. . Platelet packing density is an independent regulator of the hemostatic response to injury. J Thromb Haemost 2018;16(05):973-983

41 Xu S, Xu Z, Kim OV, Litvinov RI, Weisel JW, Alber M. Model predictions of deformation, embolization and permeability of partially obstructive blood clots under variable shear flow. J R Soc Interface 2017;14(136):14

42 Govindarajan V, Zhu S, Li R, et al. . Impact of tissue factor localization on blood clot structure and resistance under venous shear. Biophys J 2018;114(04):978-991

43 Nechipurenko DY, Receveur N, Yakimenko AO, et al. . Clot contraction drives the translocation of procoagulant platelets to thrombus surface. Arterioscler Thromb Vasc Biol 2019;39(01): 37-47

44 Trifanov P, Kaneva V, Strijhak S, et al. . Developing quasi-steady model for studying hemostatic response using supercomputer technologies. Supercomp Front Innovat 2018;5:67-72

45 Tosenberger A, Ataullakhanov F, Bessonov N, Panteleev M, Tokarev A, Volpert V. Modelling of platelet-fibrin clot formation in flow with a DPD-PDE method. J Math Biol 2016;72(03):649-681

46 Leiderman K, Fogelson AL. The influence of hindered transport on the development of platelet thrombi under flow. Bull Math Biol 2013;75(08):1255-1283

47 Khanin MA, Semenov VV. A mathematical model of the kinetics of blood coagulation. J Theor Biol 1989;136(02):127-134

48 Sorensen EN, Burgreen GW, Wagner WR, Antaki JF. Computational simulation of platelet deposition and activation: I. Model development and properties. Ann Biomed Eng 1999;27(04): 436-448

49 Purvis JE, Chatterjee MS, Brass LF, Diamond SL. A molecular signaling model of platelet phosphoinositide and calcium regulation during homeostasis and P2Y1 activation. Blood 2008;112 (10):4069-4079

50 Sveshnikova AN, Ataullakhanov FI, Panteleev MA. Compartmentalized calcium signaling triggers subpopulation formation upon platelet activation through PAR1. Mol Biosyst 2015;11(04): 1052-1060

51 Shepelyuk TO, Panteleev MA, Sveshnikova AN. Computational modeling of quiescent platelet energy metabolism in the context of whole-body glucose turnover. Math Model Nat Phenom 2016; 11:91-101

52 Obydennyi SI, Artemenko EO, Sveshnikova AN, et al. . Mechanisms of increased mitochondria-dependent necrosis in Wis-
kott-Aldrich syndrome platelets. Haematologica 2020;105(04): 1095-1106

53 Shakhidzhanov SS, Shaturny VI, Panteleev MA, Sveshnikova AN. Modulation and pre-amplification of PAR1 signaling by ADP acting via the P2Y12 receptor during platelet subpopulation formation. Biochim Biophys Acta 2015;1850(12):2518-2529

54 Martyanov AA, Balabin FA, Dunster JL, Panteleev MA, Gibbins JM, Sveshnikova AN. Control of platelet CLEC-2-mediated activation by receptor clustering and tyrosine kinase signaling. Biophys J 2020;118(11):2641-2655

55 Sveshnikova AN, Balatskiy AV, Demianova AS, et al. . Systems biology insights into the meaning of the platelet's dual-receptor thrombin signaling. J Thromb Haemost 2016;14(10):2045-2057

56 Bergmann FT, Hoops S, Klahn B, et al. . COPASI and its applications in biotechnology. J Biotechnol 2017;261:215-220

57 Burkhart JM, Vaudel M, Gambaryan S, et al. . The first comprehensive and quantitative analysis of human platelet protein composition allows the comparative analysis of structural and functional pathways. Blood 2012;120(15):e73-e82

58 Makhoul S, Walter E, Pagel O, et al. . Effects of the NO/soluble guanylate cyclase/cGMP system on the functions of human platelets. Nitric Oxide 2018;76:71-80

59 Dunster JL, Mazet F, Fry MJ, Gibbins JM, Tindall MJ. Regulation of early steps of GPVI signal transduction by phosphatases: a systems biology approach. PLOS Comput Biol 2015;11(11):e1004589

60 Dunster JL, Unsworth AJ, Bye AP, et al. . Interspecies differences in protein expression do not impact the spatiotemporal regulation of glycoprotein VI mediated activation. J Thromb Haemost 2020; 18(02):485-496

61 Martyanov AA, Morozova DS, Sorokina MA, et al. . Heterogeneity of integrin $\alpha_{\mathrm{II}} \beta_{3}$ function in pediatric immune thrombocytopenia revealed by continuous flow cytometry analysis. Int J Mol Sci 2020;21(09):21

62 Obydennyy SI, Sveshnikova AN, Ataullakhanov FI, Panteleev MA. Dynamics of calcium spiking, mitochondrial collapse and phosphatidylserine exposure in platelet subpopulations during activation. J Thromb Haemost 2016;14(09):1867-1881

63 Kotova YN, Ataullakhanov FI, Panteleev MA. Formation of coated platelets is regulated by the dense granule secretion of adenosine 5'diphosphate acting via the P2Y12 receptor. J Thromb Haemost 2008;6(09):1603-1605

64 Flamm MH, Colace TV, Chatterjee MS, et al. . Multiscale prediction of patient-specific platelet function under flow. Blood 2012; 120(01):190-198

65 Levine SN. Enzyme amplifier kinetics. Science 1966;152 (3722):651-653

66 Ratto N, Tokarev A, Chelle P, Tardy-Poncet B, Volpert V. Clustering of thrombin generation test data using a reduced mathematical model of blood coagulation. Acta Biotheor 2020;68(01):21-43

67 Zavyalova EG, Ustinov NB, Kopylov AM. Exploring the efficiency of thrombin inhibitors with a quantitative model of the coagulation cascade. FEBS Lett 2020;594(06):995-1004

68 Link KG, Stobb MT, Sorrells MG, et al. . A mathematical model of coagulation under flow identifies factor $\mathrm{V}$ as a modifier of thrombin generation in hemophilia A. J Thromb Haemost 2020;18(02):306-317

69 Siekmann I, Bjelosevic S, Landman K, Monagle P, Ignjatovic V, Crampin EJ. Mathematical modelling indicates that lower activity of the haemostatic system in neonates is primarily due to lower prothrombin concentration. Sci Rep 2019;9(01):3936

70 Mitrophanov AY, Szlam F, Sniecinski RM, Levy JH, Reifman J. Controlled multifactorial coagulopathy: effects of dilution, hypothermia, and acidosis on thrombin generation in vitro. Anesth Analg 2020;130(04):1063-1076

71 Lo K, Denney WS, Diamond SL. Stochastic modeling of blood coagulation initiation. Pathophysiol Haemost Thromb 2005;34 $(2-3): 80-90$ 
72 Balandina AN, Shibeko AM, Kireev DA, et al. . Positive feedback loops for factor $\mathrm{V}$ and factor VII activation supply sensitivity to local surface tissue factor density during blood coagulation. Biophys J 2011;101(08):1816-1824

73 Dashkevich NM, Ovanesov MV, Balandina AN, et al. . Thrombin activity propagates in space during blood coagulation as an excitation wave. Biophys J 2012;103(10):2233-2240

74 Diamond SL, Anand S. Inner clot diffusion and permeation during fibrinolysis. Biophys J 1993;65(06):2622-2643

75 Panteleev MA, Ovanesov MV, Kireev DA, et al. . Spatial propagation and localization of blood coagulation are regulated by intrinsic and protein C pathways, respectively. Biophys J 2006; 90(05):1489-1500

76 Parunov LA, Fadeeva OA, Balandina AN, et al. . Improvement of spatial fibrin formation by the anti-TFPI aptamer BAX499: changing clot size by targeting extrinsic pathway initiation. J Thromb Haemost 2011;9(09):1825-1834

77 Shibeko AM, Lobanova ES, Panteleev MA, Ataullakhanov FI. Blood flow controls coagulation onset via the positive feedback of factor VII activation by factor Xa. BMC Syst Biol 2010;4:5

78 Zhalyalov AS, Panteleev MA, Gracheva MA, Ataullakhanov FI, Shibeko AM. Co-ordinated spatial propagation of blood plasma clotting and fibrinolytic fronts. PLoS One 2017;12(07):e0180668

79 Anand M, Rajagopal K, Rajagopal KR. A model for the formation, growth, and lysis of clots in quiescent plasma. A comparison between the effects of antithrombin III deficiency and protein $C$ deficiency. J Theor Biol 2008;253(04):725-738

80 Kuharsky AL, Fogelson AL. Surface-mediated control of blood coagulation: the role of binding site densities and platelet deposition. Biophys J 2001;80(03):1050-1074

81 Ataullakhanov FI, Guria GT, Sarbash VI, Volkova RI. Spatiotemporal dynamics of clotting and pattern formation in human blood. Biochim Biophys Acta 1998;1425(03):453-468

82 Hockin MF, Jones KC, Everse SJ, Mann KG. A model for the stoichiometric regulation of blood coagulation. J Biol Chem 2002;277(21):18322-18333

83 Beltrami E, Jesty J. Mathematical analysis of activation thresholds in enzyme-catalyzed positive feedbacks: application to the feedbacks of blood coagulation. Proc Natl Acad Sci U S A 1995;92(19):8744-8748

84 Beltrami E, Jesty J. The role of membrane patch size and flow in regulating a proteolytic feedback threshold on a membrane: possible application in blood coagulation. Math Biosci 2001;172 (01):1-13

85 Kastrup CJ, Shen F, Runyon MK, Ismagilov RF. Characterization of the threshold response of initiation of blood clotting to stimulus patch size. Biophys J 2007;93(08):2969-2977

86 Panteleev MA, Balandina AN, Lipets EN, Ovanesov MV, Ataullakhanov FI. Task-oriented modular decomposition of biological networks: trigger mechanism in blood coagulation. Biophys J 2010;98(09):1751-1761

87 Diamandis P, Wildenhain J, Clarke ID, et al. . Chemical genetics reveals a complex functional ground state of neural stem cells. Nat Chem Biol 2007;3(05):268-273

88 Kuprash AD, Shibeko AM, Vijay R, et al. . Sensitivity and robustness of spatially dependent thrombin generation and fibrin clot propagation. Biophys J 2018;115(12):2461-2473
89 Mitrophanov AY, Wolberg AS, Reifman J. Kinetic model facilitates analysis of fibrin generation and its modulation by clotting factors: implications for hemostasis-enhancing therapies. Mol Biosyst 2014;10(09):2347-2357

90 Mitrophanov AY, Szlam F, Sniecinski RM, Levy JH, Reifman J. A step toward balance: thrombin generation improvement via procoagulant factor and antithrombin supplementation. Anesth Analg 2016;123(03):535-546

91 Sinauridze EI, Gorbatenko AS, Seregina EA, Lipets EN, Ataullakhanov FI. Moderate plasma dilution using artificial plasma expanders shifts the haemostatic balance to hypercoagulation. Sci Rep 2017;7(01):843

92 Brummel-Ziedins KE, Gissel M, Neuhaus J, et al; INSIGHT SMART, ESPRIT Study Groups. In silico thrombin generation: Plasma composition imbalance and mortality in human immunodeficiency virus. Res Pract Thromb Haemost 2018;2(04):708-717

93 Gupta S, Bravo MC, Heiman M, et al. . Mathematical model of thrombin generation and bleeding phenotype in Amish carriers of Factor IX:C deficiency vs. controls. Thromb Res 2019; 182:43-50

94 Brummel-Ziedins KE, Whelihan MF, Gissel M, Mann KG, Rivard GE. Thrombin generation and bleeding in haemophilia A. Haemophilia 2009;15(05):1118-1125

95 Brummel-Ziedins KE, Vossen CY, Butenas S, Mann KG, Rosendaal FR. Thrombin generation profiles in deep venous thrombosis. J Thromb Haemost 2005;3(11):2497-2505

96 Brummel-Ziedins K, Vossen CY, Rosendaal FR, Umezaki K, Mann KG. The plasma hemostatic proteome: thrombin generation in healthy individuals. J Thromb Haemost 2005;3(07):1472-1481

97 Chelle P, Morin C, Montmartin A, Piot M, Cournil M, Tardy-Poncet B. Evaluation and calibration of in silico models of thrombin generation using experimental data from healthy and haemophilic subjects. Bull Math Biol 2018;80(08):1989-2025

98 Duarte RCF, Ferreira CN, Rios DRA, Reis HJD, Carvalho MDG. Thrombin generation assays for global evaluation of the hemostatic system: perspectives and limitations. Rev Bras Hematol Hemoter 2017;39(03):259-265

99 van Veen JJ, Gatt A, Makris M. Thrombin generation testing in routine clinical practice: are we there yet? $\mathrm{Br} J$ Haematol 2008; 142(06):889-903

100 Liang Y, Woodle SA, Shibeko AM, Lee TK, Ovanesov MV. Correction of microplate location effects improves performance of the thrombin generation test. Thromb J 2013;11(01):12

101 Bannish BE, Keener JP, Fogelson AL. Modelling fibrinolysis: a 3D stochastic multiscale model. Math Med Biol 2014;31(01):17-44

102 Bannish BE, Chernysh IN, Keener JP, Fogelson AL, Weisel JW. Molecular and physical mechanisms of fibrinolysis and thrombolysis from mathematical modeling and experiments. Sci Rep 2017;7(01):6914

103 Shibeko AM, Woodle SA, Mahmood I, Jain N, Ovanesov MV. Predicting dosing advantages of factor VIla variants with altered tissue factor-dependent and lipid-dependent activities. J Thromb Haemost 2014;12(08):1302-1312

104 Piebalgs A, Gu B, Roi D, Lobotesis K, Thom S, Xu XY. Computational simulations of thrombolytic therapy in acute ischaemic stroke. Sci Rep 2018;8(01):15810 